\title{
Prestação de serviços odontológicos em instituições federais públicas de ensino superior e a integração com a rede de atenção à saúde
}

\author{
Fabiane Alves Farias Guimarães*; Ana Lúcia Schaefer Ferreira de Mello**
}

* Mestre em Odontologia, área de concentração em Saúde Coletiva, UFSC

** Doutora em Odontologia, Professora dos Programas de PósGraduação em Odontologia e em Enfermagem, UFSC

Recebido em 30/01/2017. Aprovado em 03/06/2017.

\begin{abstract}
RESUMO
O estudo objetivou analisar os serviços prestados pelas Instituições Federais de Ensino Superior (IFES) com curso de graduação em Odontologia no sul do Brasil, compreendendo a maneira como se organizam e a integração com a Rede de Atenção à Saúde (RAS). Trata-se de um estudo exploratório, descritivo e analítico, de abordagem qualitativa, utilizando técnicas de observação, análise documental e entrevistas semiestruturadas, realizadas no período de junho a outubro de 2014. A plena integração das clínicas odontológicas das IFES com a RAS ainda é um desafio a ser superado, o que vem comprometendo a efetivação dos princípios do Sistema Único de Saúde no atendimento odontológico dos pacientes e repercutindo na formação profissional em Odontologia. Faz-se necessário consolidar a relação das IFES com o sistema público de saúde para que as clínicas odontológicas possam, como um ponto de atenção, efetivamente fazer parte da RAS.
\end{abstract}

Descritores: Clínicas Odontológicas. Estudantes de Odontologia. Recursos Humanos em Odontologia. Universidades. Assistência à Saúde.

\section{INTRODUÇÃO}

A inserção dos profissionais de Odontologia no setor público de saúde resultou em preocupações referentes ao perfil dos cirurgiões dentistas diante de novos cenários de prática profissional $^{1}$. No âmbito do ensino superior em saúde, vêm sendo debatidas novas maneiras de trabalhar o conhecimento dentro de uma perspectiva crítica e reflexiva, centrada na integralidade, acompanhando as políticas públicas nas áreas de Educação e Saúde ${ }^{2}$. As Diretrizes Curriculares Nacionais $(\mathrm{DCN})^{3}$ mostraram um caminho a ser percorrido para que a formação na área da saúde esteja de acordo com as necessidades 
da população, propondo às Instituições de Ensino Superior (IES) do Brasil o desafio de formar cirurgiões dentistas em sintonia com o sistema de saúde vigente ${ }^{4}$. A necessidade de modificações nos cursos de graduação enfrenta alguns obstáculos. Assim, novos caminhos estão sendo buscados para responder ao desafio proposto, incluindo a implementação de novos projetos pedagógicos nos cursos e mudanças curriculares 5 .

$\mathrm{O}$ distanciamento entre os mundos acadêmico e o da prestação real dos serviços de saúde vem sendo apontado como um dos responsáveis pela crise do setor saúde ${ }^{6}$. Esse contexto revela a necessidade de adequação entre a formação dos profissionais e vida real, entre teoria e prática, entre teorização e aplicabilidade de fato do conhecimento ${ }^{7,8}$.

As faculdades/cursos de Odontologia de instituições públicas são prestadores de serviços odontológicos para a população e fazem parte do Sistema Único de Saúde (SUS). Assim, a prática clínica que acontece em seus ambientes deve estar de acordo com um novo modelo pedagógico que priorize tanto a qualidade técnica quanto a relevância social, contemplando aspectos como a produção de subjetividade, produção de habilidades técnicas e de pensamento, bem como o adequado conhecimento do SUS ${ }^{9}$.

Buscando ampliar a oferta e o acesso da população às ações e serviços de saúde bucal, qualificar os serviços, promover a cooperação entre os gestores do SUS e as IES com curso de graduação em Odontologia à rede pública de serviços de saúde bucal, foi criado, por meio de uma parceria entre os ministérios da Saúde e da Educação, o componente GraduaCEO, no âmbito da Política Nacional de Saúde Bucal ${ }^{10}$. Dessa maneira as IES com curso de graduação em Odontologia participam da Rede de Atenção à Saúde (RAS), que é descrita como arranjos organizativos de ações e serviços de saúde, de diferentes densidades tecnológicas, que buscam garantir a integralidade do cuidado por meio de sistemas integrados de apoio técnico, logístico e de gestão $^{11}$

Assim, os serviços prestados pelas IES com curso de graduação em Odontologia, no contexto das diretrizes e princípios do SUS e das mudanças curriculares e sua relação com a RAS é o foco deste artigo. $\mathrm{O}$ argumento se fundamenta nas consequências prejudiciais geradas ao sistema de saúde e à saúde da população devido à inadequação da formação em saúde ao longo do tempo. Dentre as características que contribuíram para a inadequação da formação dos profissionais de saúde está a fragmentação e a descontextualização de conteúdos e a centralização do professor especialista no processo ensino aprendizagem ${ }^{12}$. A adoção pelos cursos e faculdades de Odontologia de referenciais teóricos fundamentados no conceito ampliado de saúde, evoluindo de um modelo assistencial centrado na doença e baseado no atendimento a quem procura, para um modelo de atenção integral à saúde tanto no que se refere ao ensino quanto à prestação de serviços odontológicos para a população é um desafio que deve ser superado 9,10

Nesse contexto, o objetivo do estudo foi analisar a integração dos serviços odontológicos com a Rede de Atenção à Saúde (RAS-SUS), prestados por três Instituições Federais de Ensino Superior (IFES) com curso de graduação em Odontologia do sul do Brasil.

\section{MÉTODO}

Trata-se de um estudo exploratório, descritivo e analítico, de abordagem qualitativa. As estratégias metodológicas utilizadas compreenderam observação, análise documental e entrevistas semiestruturadas. $\mathrm{O}$ estudo foi conduzido em três IFES públicas do sul do Brasil, durante o período de junho a outubro de 2014. Estas foram selecionadas intencionalmente por critério de proximidade do local de origem dos pesquisadores. A população de 
estudo foi selecionada intencionalmente $\mathrm{e}$ priorizando os indivíduos com maior envolvimento com o objeto de investigação, utilizando a técnica snowball.

Inicialmente foram entrevistados gestores municipais e gestores acadêmicos; estes últimos indicavam docentes, servidores técnicoadministrativos e discentes. Portanto, a amostra foi constituída por gestores da área de saúde bucal dos municípios em que as IFES estão inseridas, gestores acadêmicos, docentes, discentes e servidores do setor de admissão de pacientes das IFES participantes do estudo. Para delimitar o número de entrevistados foi utilizado o procedimento de saturação teórica dos dados.

Foram realizadas vinte e quatro entrevistas, sendo oito na IFES 1 , nove na IFES 2 e sete na IFES 3. Em relação à característica do entrevistado, sete entrevistas foram realizadas com docentes, nove com discentes, três com servidores responsáveis pelo setor de admissão de pacientes, duas com gestores acadêmicos das IFES e três com gestores da área de saúde bucal dos municípios em que as IFES se inserem.

As entrevistas foram gravadas em um gravador de áudio e tiveram durações bastante variadas: entre quinze minutos e uma hora e meia. As entrevistas com discentes foram as mais curtas e com os demais participantes, mais longas. Após a transcrição as entrevistas não retornaram para os participantes para comentários ou correções.

Para a análise documental, foram utilizados os projetos político pedagógico dos cursos, programas das disciplinas clínicas ou que prestam assistência odontológica à população, currículos com ementas e o documento que implementa a rede docente assistencial de um município. Esses documentos foram obtidos por meio do acesso aos endereços eletrônicos das instituições participantes do estudo.

A observação direta não participante foi escolhida com o propósito de permitir acompanhar e registrar os movimentos, os discursos e as ações dos diversos atores sociais envolvidos. Para o registro dos dados obtidos nas observações, foi utilizado um diário de campo. Essa observação aconteceu no interior das IFES participantes do estudo, incluindo salas de espera, setor de admissão de pacientes, secretaria dos serviços que prestam atendimento direto à população (serviços de apoio diagnóstico e estomatologia), clínicas odontológicas das IFES e Centro de Especialidades Odontológicas que operam nas IFES.

Os dados obtidos por meio da realização das entrevistas, análise documental e observação foram analisados separadamente. A metodologia de análise de conteúdo proposta por Laurence Bardin norteou a análise dos dados oriundos das entrevistas individuais ${ }^{13}$. Seguindo o método de análise de conteúdo, foi realizada a leitura de todo o material transcrito oriundo das entrevistas individuais. Foram destacadas as partes principais de cada sentença para que as informações pudessem ser codificadas. Com base na codificação das entrevistas foram elaboradas as categorias principais e as subcategorias.

Os dados obtidos nas IFES foram analisados em conjunto, buscando não destacar as especificidades de cada instituição. As informações obtidas a partir da análise documental foram utilizadas para conhecer o histórico, o projeto político pedagógico, o currículo, os programas das disciplinas clínicas e que prestam assistência à população nas IFES participantes do estudo, permitindo realizar a descrição dessas IFES e uma aproximação ao contexto em estudo. $\mathrm{O}$ material oriundo da observação realizada subsidiou a condução das entrevistas individuais assim como serviu de suporte para análise destas e discussão dos dados.

A pesquisa foi aprovada pelo Comitê de Ética e Pesquisa com Seres Humanos da Universidade Federal de Santa Catarina, sob o Parecer $n^{\circ}$ 711.411. Os entrevistados foram convidados, ouviram os objetivos do estudo, concordaram em participar e assinaram o Termo de Consentimento Livre e 
Esclarecido.

\section{RESULTADOS E DISCUSSÃO}

Os resultados são apresentados a seguir, acompanhados da discussão com a literatura, segundo as categorias analisadas (quadro 1).

\section{O acesso da população aos serviços odonto- lógicos em IFES com curso de graduação em Odontologia}

A demanda espontânea é uma das formas de acesso aos serviços odontológicos ofertados pelas IFES, em que o atendimento é buscado diretamente em um setor de admissão de pacientes denominado "Triagem". Em algumas situações, o paciente é avaliado neste setor por cirurgiões dentistas ou acadêmicos para, posteriormente, ser encaminhado para as clínicas ou para o atendimento de urgência. Em outras situações, o paciente relata as suas queixas e as necessidades de tratamento, informa seus dados para contato e essas informações irão compor uma lista de espera a partir da qual serão contatados posteriormente para o atendimento. Os pacientes podem residir em qualquer área da cidade ou mesmo em outras cidades, não havendo, portanto, uma área adscrita.

Algumas IFES realizaram contratualização com o SUS e o paciente não paga diretamente pelo atendimento, exceto os serviços que precisam ser realizados externamente, por exemplo, os Laboratórios de Prótese Dentária; em outras IFES o serviço ofertado por demanda espontânea não acontece integrado ao SUS e o paciente precisa pagar tanto uma taxa por consulta quanto por serviços externos.

O acesso por demanda espontânea dificulta a integração das clínicas odontológicas das IFES com a Rede de Atenção à Saúde Bucal (RASB). Esse tipo de acesso é considerado inadequado frente à Portaria GraduaCEO, que prevê o preenchimento das vagas das clínicas odontológicas da graduação em Odontologia por meio do sistema de regulação municipal ou estadual ${ }^{10}$. As IFES participantes do estudo ainda não haviam aderido ao GraduaCEO quando da coleta de dados, mas essa modalidade de acesso precisa ser repensada.

Existe um mecanismo de encaminhamento formal entre a RAS municipal e as IFES que acontece para os CEO que operam nas IFES e, excepcionalmente, para clínicas chamadas de "baixa complexidade". Essa modalidade de acesso, organizado pela regulação municipal, facilita a integração das clínicas odontológicas das IFES com a RASB. Assim, conforme preconizado pelas diretrizes para organização da RAS do SUS, a Atenção Primária à Saúde coordena o cuidado e ordena a rede, incluindo as clínicas odontológicas das IFES como um dos pontos de atenção da RAS ${ }^{11}$.

Para os serviços que têm custo para o paciente, o município não realiza um encaminhamento formal. Nesses casos, uma possibilidade é que os profissionais da RASB municipal realizem um laudo técnico e, de posse desse documento, o paciente pode buscar o atendimento na IFES por demanda espontânea.

O paciente não terá garantia de vaga para o atendimento nas clínicas odontológicas da IFES. Havendo disponibilidade, o paciente será encaminhado para atendimento, caso contrário ele será orientado a retornar à IFES nos períodos de seleção do setor de admissão de pacientes.

Essa situação destaca uma realidade que foi evidenciada no estudo de Gonçalves e Verdi ${ }^{14}$ : os pacientes que buscam um atendimento nas clínicas odontológicas das IES podem passar por diversas situações que tornam o caminho até o atendimento permeado por atalhos e desvios.

As listas de espera são organizadas por disciplina, procedimento ou clínica odontológica. Algumas vezes os pacientes ficam durante longos períodos na lista de espera e quando são agendados a necessidade de tratamento já sofreu alteração, sendo necessário uma reavaliação e talvez um reencaminhamento para outra clínica ou disciplina. 
Quadro 1. Categorias e subcategorias elaboradas a partir da análise dos dados.

\begin{tabular}{|c|c|}
\hline Categorias & Subcategorias \\
\hline $\begin{array}{l}\text { O acesso da população aos serviços } \\
\text { odontológicos em IFES com curso de } \\
\text { graduação em Odontologia }\end{array}$ & $\begin{array}{l}\text { - O acesso por demanda espontânea } \\
\text { - O acesso via encaminhamento formal } \\
\text { - O acesso via indicação ou encaminhamento informal } \\
\text { - Mecanismos de triagem e elaboração das listas de espera } \\
\text { - Acesso aos serviços de patologia, radiologia e projetos de extensão }\end{array}$ \\
\hline $\begin{array}{l}\mathrm{O} \text { agendamento de pacientes para } \\
\text { atendimento odontológico }\end{array}$ & $\begin{array}{l}\text { - Ordenação da chamada dos pacientes } \\
\text { - Mecanismos de agendamento das consultas } \\
\text { - O agendamento no Centro de Especialidades Odontológicas } \\
\text { - O agendamento de pacientes que não entraram formalmente no } \\
\text { atendimento da faculdade } \\
\text { - Perspectivas futuras para o agendamento dos pacientes }\end{array}$ \\
\hline $\begin{array}{l}\text { Fluxos internos dos pacientes nas } \\
\text { clínicas das IFES com curso de } \\
\text { graduação em Odontologia }\end{array}$ & $\begin{array}{l}\text { - Características das clínicas da faculdade e da integração entre elas } \\
\text { - Encaminhamentos entre as clínicas/disciplinas através do setor de } \\
\text { triagem/recepção } \\
\text { - Encaminhamentos entre as clínicas/disciplinas através dos professores } \\
\text { - Encaminhamentos entre as clínicas/disciplinas através dos alunos } \\
\text { - Clínicas/disciplinas que não funcionam integradas às outras } \\
\text { - Integração entre as clínicas da graduação e serviços da pós-graduação } \\
\text { - Os serviços de urgência odontológica }\end{array}$ \\
\hline $\begin{array}{l}\text { Funcionamento dos Centros de } \\
\text { Especialidades Odontológicas nas IFES }\end{array}$ & $\begin{array}{l}\text { - Características dos Centros de Especialidades Odontológicas que } \\
\text { operam nas IES } \\
\text { - Referência e contrarreferência nos Centros de Especialidades } \\
\text { Odontológicas que operam nas IES }\end{array}$ \\
\hline $\begin{array}{l}\text { Integração da IFES com a rede pública } \\
\text { de saúde municipal }\end{array}$ & $\begin{array}{l}\text { - Aspectos históricos da integração dos Cursos/Faculdades em IES com a } \\
\text { rede de atenção à saúde bucal municipal } \\
\text { - Integração das clínicas odontológicas dos cursos/faculdades com a rede } \\
\text { municipal } \\
\text { - Integração das unidades básicas de saúde com os cursos/faculdades } \\
\text { - Parcerias entre faculdade e rede municipal } \\
\text { - Registros e prontuários } \\
\text { - Perspectivas futuras para integração da faculdade com a rede municipal }\end{array}$ \\
\hline \multicolumn{2}{|l|}{ Interação com outros serviços/cursos } \\
\hline $\begin{array}{l}\text { Efeitos da organização dos serviços } \\
\text { odontológicos prestados por IFES com } \\
\text { curso de graduação em Odontologia no } \\
\text { processo ensino-aprendizagem }\end{array}$ & $\begin{array}{l}\text { - As mudanças ocorridas no currículo e na rede municipal } \\
\text { - A Portaria GraduaCEO }\end{array}$ \\
\hline
\end{tabular}

De acordo com a Portaria No 4.279, que trata da organização da $\operatorname{RAS}^{11}$, a lista de espera normatiza o uso dos serviços de maneira transparente, estabelecendo critérios de ordenamento segundo necessidade e riscos visando equilibrar a oferta e a demanda. No caso das IFES, a oferta está diretamente relacionada à necessidade de aprendizado dos acadêmicos. Contudo, muitas vezes essa oferta não está de acordo com a demanda.

$\mathrm{O}$ acesso aos serviços de apoio diagnóstico (patologia, radiologia) e aos projetos de extensão frequentemente acontece distintamente das clínicas e disciplinas. Esses serviços têm uma lista de espera separada e o atendimento é ofertado direto à 
população, sem envolvimento maior do setor de admissão de pacientes.

\section{O agendamento de pacientes para atendi- mento odontológico}

As listas de espera são organizadas de acordo com a ordem de chegada. Quando uma vaga fica disponível, é realizado o contato com o paciente. Contudo, diversas situações podem impedir que o próximo paciente da lista de espera seja efetivamente atendido: custos das ligações telefônicas, o paciente não atender ao telefonema, o número telefônico dos dados cadastrais estar errado, o paciente já ter procurado outro serviço de saúde para ser atendido ou $\mathrm{o}$ paciente não poder comparecer no horário disponibilizado pela IFES.

É possível perceber a dificuldade no acesso no que se refere à comodidade: a dificuldade de contato entre a IFES e o paciente, assim como os horários de atendimento que não são convenientes, especialmente às pessoas que trabalham no horário comercial, quando funcionam a maior parte das clínicas ${ }^{11}$.

Alguns estudos ${ }^{15-17}$ destacaram que os serviços de saúde ofertados pelas clínicas odontológicas de IES são predominantemente utilizados por mulheres, possivelmente por sua menor inserção no mercado formal de trabalho, facilitando $\mathrm{o}$ agendamento das consultas nos horários disponibilizados pelas IES.

$\mathrm{O}$ agendamento a partir de pacientes encaminhados pelo município facilita a inte-gração entre as clínicas odontológicas das IFES e a RASB municipal. Atualmente esse mecanismo está acontecendo apenas para as clínicas de "baixa complexidade", mas com a implantação do GraduaCEO é possível expandir esses encaminhamentos para as demais $\operatorname{clínicas}^{10}$. Para os procedimentos mais complexos (próteses, implantes, aparelhos ortodônticos) há uma lista de espera própria do setor de triagem da IFES que opera por demanda espontânea.
Ao ficar disponível uma vaga para atendimento, estudantes agendam pacientes que não passaram pelo processo formal de acesso aos serviços. Incluem na sua agenda amigos, familiares, pessoas que têm necessidades de tratamento que ele precisa realizar por questões relacionadas ao seu aprendizado.

Nesses casos a vaga disponível não será preenchida por quem está na lista de espera e passou pelo processo formal de acesso aos serviços da IFES. Em algumas IFES isso é permitido, desde que os professores responsáveis pela disciplina autorizem e justifiquem porque um paciente de fora da lista de espera será atendido. Contudo, muitas vezes essa justificativa não é apresentada. Essa realidade vai ao encontro do trabalho de Gonçalves e Verdi ${ }^{14}$, que revelou o desrespeito ao princípio da autonomia dos que esperam por uma vaga nas clínicas da IES pesquisada, já que o acesso privilegia algumas pessoas.

Uma vez que a oferta de vagas nas clínicas odontológicas das IES está relacionada com a necessidade dos estudantes em realizar as atividades práticas necessárias para a sua formação, é importante que esses procedimentos sejam identificados previamente e que a oferta dessas vagas aconteça para os pacientes que passaram pelo processo formal de acesso, seja por demanda espontânea ou encaminhando pela RASB municipal. As listas de espera promovem uma transparência nesse processo, sendo necessário respeitar a normatização do uso dos serviços ${ }^{11}$.

A falta de informatização nas clínicas das IES foi apontada como um problema a ser superado, uma vez que isso gera uma grande quantidade de papéis e formulários a serem preenchidos, além da possibilidade desses documentos serem perdidos. A informatização das clínicas e do setor de admissão de pacientes facilitará o processo de agendamento, cadastro e armazenamento de prontuários, possibilitando até mesmo que esse sistema seja integrado ao município, como é preconizado para 
organização das RAS no SUS ${ }^{11}$.

\section{Fluxos internos dos pacientes nas clínicas das IFES com curso de graduação em Odontologia}

Há situações em que as clínicas odontológicas integram diferentes disciplinas, sendo separadas apenas por níveis de complexidade dos procedimentos realizados: clínicas de baixa, média e alta complexidade. Dessa forma os estudantes recebem os pacientes na primeira clínica integrada e vão sendo acompanhados por este estudante ao longo das próximas clínicas. Assim, a maior parte dos encaminhamentos internos acaba não necessitando acontecer.

Albuquerque et al. $^{18}$ evidenciaram que o currículo integrado valoriza o espaço de articulação entre ensino, serviço e comunidade, permitindo ao discente um processo de aprendizagem crítico e reflexivo sobre as suas ações e a realidade da localidade em que ele está inserido. Assim, como o estudante vai acompanhando o paciente ao longo das clínicas, aspectos como a integralidade e a longitudinalidade do cuidado podem ser percebidos pelo estudante.

Muitas vezes os encaminhamentos entre as diversas clínicas/disciplinas dentro da IFES acontecem de maneira desorganizada. $\mathrm{O}$ acompanhamento do paciente ao longo do tratamento também é relatado como um problema, pois, se o paciente é encaminhado para outra clínica, o estudante e o professor orientador não têm conhecimento do tratamento realizado.

No estudo realizado por Ferreira et al. ${ }^{19}$, estudantes de Odontologia apontaram que problemas relacionados ao processo organizacional no interior das IES, como horários, burocracia, número insuficiente de professores, falta de informatização, falta de pacientes e falta de materiais, têm grande influência sobre seus desempenhos. É importante superar esses problemas a fim de garantir que essas questões relacionadas ao processo de organização da IES não tenham impactos negativos na formação dos estudantes e no atendimento prestados aos usuários das clínicas.

$\mathrm{O}$ encaminhamento entre as clínicas/ disciplinas pode acontecer por meio do setor de triagem. O paciente é colocado na lista de espera da disciplina para a qual foi encaminhado. Sempre se prioriza o paciente que já está sendo atendido, portanto os pacientes que são encaminhados de uma clínica para outra têm prioridade em relação aos que estão acessando os serviços pela primeira vez.

O encaminhamento entre as clínicas/ disciplinas pode acontecer via professores. Nesse caso, o estudante encaminha o paciente que já está em atendimento em uma clínica/disciplina para o professor responsável de outra clínica/ disciplina sem passar pelo setor de triagem, uma vez que já se tem conhecimento do plano de tratamento e todas as informações dos procedimentos já realizados ficam registrados no prontuário do paciente.

Se o paciente precisa ser encaminhado de uma clínica/disciplina para outra e o estudante conhece algum colega que está nesta outra clínica é realizado o encaminhamento entre esses estudantes sem passar pelo setor de triagem. Os estudantes relatam que é positivo quando isso acontece, pois, o paciente não precisará aguardar na lista de espera da outra clínica/disciplina e há um comprometimento por parte do estudante em atender esse paciente.

Tanto as DCN para os cursos de graduação em Odontologia quanto as diretrizes para organização da RAS no SUS destacam a importância da integralidade da atenção prestada aos usuários $^{3,11}$. Esse princípio deve ser considerado também na prestação de serviços que ocorre no ambiente das clínicas odontológicas das IFES.

De acordo com Ferreira et al..$^{19}$, a realização de planos de tratamento completos é um ponto fundamental de uma clínica integrada, auxiliando na superação da segmentação do cuidado ofertado nas clínicas odontológicas de IES.

Toassi et al. ${ }^{5}$ acreditam que a dificuldade dos docentes em realizar a integração curricular seja 
uma justificativa para essa fragilidade na integração. Essa situação pode ser devido à insuficiente capacitação ou desconhecimento do professor em relação a metodologias de ensino-aprendizagem que sejam propícias à essa integração.

As IFES também ofertam serviços de urgência à população. Esses serviços funcionam junto às disciplinas obrigatórias, eletivas e como estágio.

\section{Funcionamento dos Centros de Especia- lidades Odontológicas nas IFES}

Os CEO que operam nas IFES são resultado de convênio entre a universidade e as Secretarias Estadual ou Municipal de Saúde para a prestação de serviços especializados.

A maneira como os estudantes atuam no CEO é diversificada: pode ser um campo de estágio obrigatório ou optativo; para os estudantes de graduação, nos semestres mais avançados do curso, e de pós-graduação. Esses CEO são diferenciados, pois são tanto uma unidade prestadora de serviços quanto um local de ensino. Por vezes, os estudantes da graduação atendem em todas as especialidades ofertadas pelo CEO e contam com o auxílio e supervisão de professores da IFES e de profissionais contratados.

Os CEO que operam nas IFES fazem parte da RAS do SUS e recebem um financiamento do SUS para a realização das atividades. Assim como os demais CEO, têm uma meta de procedimentos a cumprir por mês ${ }^{20}$.

Os CEO que operam nas IFES recebem os pacientes referenciados da rede municipal para a realização de tratamento em determinada especialidade e, após a conclusão do tratamento, é realizada a contrarreferência para a UBS de origem, que é responsável pela manutenção e acompanhamento.

Atualmente, esses CEO são a principal forma de integração entre a RASB e os serviços prestados nas clínicas odontológicas das IFES, compondo a
RASB como um ponto de atenção secundária e as formas de acesso, referência e contrarreferência acontecem de acordo com o preconizado para a organização das RAS no SUS ${ }^{11}$.

\section{Integração da IFES com a rede pública de saúde municipal}

Os encaminhamentos formais da UBS se dão apenas para o CEO da faculdade. O serviço ofertado pelo CEO fica restrito ao atendimento em especialidade e posteriormente o paciente retorna para UBS. Entretanto, indicações são feitas para alguns projetos específicos, dentro da faculdade, que são referência em atendimento como trauma de dente decíduo, trauma de dente permanente, diagnóstico bucal, entre outros.

As faculdades têm dado um apoio importante para a rede municipal na organização de algumas capacitações, cursos na modalidade à distância, suporte à programação de educação permanente, especialmente na área de Saúde Coletiva. A rede municipal se propõe a ser um campo de estágio, recebendo estudantes nas UBS e também em outros pontos de atenção da rede. Os professores acompanham os estudantes de graduação, supervisionando, juntamente com os preceptores da rede, as atividades desenvolvidas. Segundo Toassi et $a l .^{21}$, esses estágios que possibilitam a presença dos estudantes no serviço têm a capacidade de aproximar a universidade, o serviço e a população.

Como as IFES com curso de graduação em Odontologia ainda não estão formalizadas como pertencentes à rede pública de atenção à saúde bucal, a universidade não pode encaminhar pacientes para outros pontos da rede além da atenção básica. A universidade ainda não tem uma área geográfica definida, uma população adscrita sob sua responsabilidade, e não participa do sistema de regulação como uma unidade solicitante, ou seja, participa apenas como uma unidade executante. Ainda é necessário realizar as pactuações entre a universidade e os gestores do SUS sobre como será 
a organização da inserção das clínicas odontológicas das IFES na RASB.

\section{Interação com outros serviços/cursos}

Formalmente não existe um atendimento multiprofissional no âmbito da graduação nas IFES. Contudo, existem alguns relatos relacionados a atividades multiprofissionais, corroborando com o estudo de Ferreira et al. ${ }^{19}$ que mostrou a inexistência de um trabalho multiprofissional nas clínicas da IFES estudada. Esses autores destacam a importância de diversificar os cenários de prática, incluindo atividades extramuros a fim de propiciar aos estudantes de graduação em Odontologia a experiência de atuação em equipe multiprofissional.

$\mathrm{Na}$ clínica odontológica infantil e na de pacientes com necessidades especiais, percebeu-se a necessidade da presença de outros profissionais, entretanto os problemas são resolvidos de maneira mais pontual, sem um protocolo formal.

Efeitos da organização dos serviços odontológicos prestados por IFES com curso de graduação em Odontologia no processo ensino-aprendizagem

As clínicas odontológicas das IFES se dividem entre o atendimento à população e o ensino dos acadêmicos. Existe um questionamento relacionado aos aspectos éticos envolvidos com essa prática de agendar os pacientes de acordo com a necessidade do estudante e não por ordem de chegada ou necessidade do usuário. Eventualmente, por interesse didático, é permitido que o estudante faça o agendamento de um paciente que não passou pelo sistema formal de acesso aos serviços da faculdade.

Nas IFES com curso de graduação em Odontologia há um forte direcionamento em relação ao SUS, superando uma visão antiga de que os serviços prestados são desconectados do SUS. No currículo vigente, está previsto estágios dos estudantes de graduação na rede para que os estudantes tenham contato e conhecimento do funcionamento do serviço público e, assim, estejam mais preparados para o trabalho no $\mathrm{SUS}^{3}$.

Existe um movimento favorável das IFES para aderir ao GraduaCEO, uma parceria entre os ministérios da Saúde e da Educação com o propósito de ampliar a oferta e o acesso da população às ações e serviços de saúde bucal, qualificar os serviços, ampliar a cooperação entre os gestores do SUS e as IES e integrar as clínicas odontológicas das IES com curso de graduação em Odontologia à rede pública de serviços de saúde bucal. Essa Portaria foi divulgada recentemente e é necessário realizar pactuações sobre como esse processo de mudança vai se configurar ${ }^{10}$.

O estudante terá um ganho na sua formação entendendo melhor como funciona o SUS, pois a Universidade ainda está um pouco afastada deste. Isso vai ao encontro do que foi evidenciado por Freitas, Calvo e Lacerda $^{21}$ ao afirmar que a integração entre o sistema público e os cursos de graduação em Odontologia ainda não está consolidada nas orientações de reestruturação curricular dos cursos.

A implantação do GraduaCEO envolvendo representantes da Universidade e gestores do SUS é um processo que está em andamento. As questões referentes à estrutura física, recursos humanos, organização do funcionamento interno e equipamentos devem ser ajustadas para que as IFES possam aderir a essa iniciativa.

\section{CONCLUSÕES}

A integração entre as clínicas odontológicas das IFES e a RASB está em um momento de transitoriedade. $\mathrm{O}$ acesso da população aos serviços ofertados pode acontecer por demanda espontânea ou por referenciamento. As listas de espera são organizadas por ordem de chegada, não sendo aplicados outros critérios de prioridade. Os agendamentos dos pacientes referenciados da RAS 
são realizados pelo setor de regulação municipal. A referência da RASB municipal acontece para os CEO que operam nas IFES e, em alguns casos, para as clínicas de baixa complexidade.

A integração entre as clínicas odontológicas se dá pela sua própria organização. Os achados evidenciaram problemas relacionados aos fluxos internos, que precisam ser melhor estabelecidos e divulgados. Essa realidade traz consequências prejudiciais ao paciente, que tem seu atendimento fragmentado e, muitas vezes, não consegue finalizar seu tratamento, prejudicando a integralidade e longitudinalidade do cuidado à saúde bucal.

A partir da instituição da portaria GraduaCEO nas IES, estimam-se que mudanças estarão em curso. O GraduaCEO pode, assim, promover uma melhor a relação das IES com a RAS pela definição clara de papéis dos pontos de atenção, pela formalização dos fluxos de atendimento, pela possibilidade de complementação por parte das IES da oferta de serviços, os quais o nível municipal tem dificuldades em oferecer, e pelo estimulo ao alinhamento dos serviços odontológicos prestados pelas IES aos princípios do SUS. Nesse sentido, será importante a realização de novos estudos sobre essa temática quando as adesões a esta iniciativa já tenham ocorrido.

\section{ABSTRACT \\ Provision of dental services in public dental schools and integration with the health care network}

The purpose of this study was to analyze the services provided by federal public dental schools (DS) in southern Brazil, particularly the organization and the integration with the Health Care Network (HCN). This is an exploratory, descriptive and analytical study with a qualitative approach using observation techniques, documentary analysis and semi-structured interviews, carried out from June to October 2014. The full integration of the dental services with the HCN is still a challenge to be overcome, which has compromised the effectiveness of the national health system principles and has consequences on professional training in dentistry. It is necessary to consolidate the relationship of the DS with the public health system so that dental services provided by DS can, effectively, be part of the HCN.

Descriptors: Dental Clinics. Students, Dental. Dental Staff. University. Delivery of Health Care.

\section{REFERÊNCIAS}

1. Dias HS. A implementação da política de reorientação da formação em odontologia: dependência de trajetória e estímulos institucionais na UFBA [dissertação]. Rio de Janeiro: Escola Nacional de Saúde Pública Sérgio Arouca; 2011.

2. Scorzoni MF, Bueno SMV, Coscrato G. O currículo e as implicações dos novos paradigmas educacionais na formação do enfermeiro. Saúde Transform Soc. 2013; 4(1):11-5.

3. Brasil. Conselho Nacional de Educação. Câmara de Educação Superior. Resolução CNE/CES 3, de 19 de fevereiro de 2002. Institui Diretrizes Curriculares Nacionais do Curso de Graduação em Odontologia. DiárioOficial da União, Brasília, 4 de março de 2002.

4. Moysés ST, Kriger L, Moysés SJ. Saúde Bucal das Famílias - Trabalhando com Evidências. São Paulo: Artes Médicas, 2008. p. 268-276.

5. Toassi RFC, Stobaus CD, Mosquera JJM, Moyses SJ. Currículo integrado no ensino de Odontologia: novos sentidos para a formação na área da saúde. Interface Comun Saúde Educ. 2012;16(41):529-42.

6. Brasil. Ministérios da Educação e da Saúde. Programa Nacional de Reorientação da Formação Profissional em Saúde PRÓ-SAÚDE. Diário Oficial da União, Brasília, 17 de novembro de 2005.

7. Mattos RA. A integralidade na prática (ou sobre a prática da integralidade). Cad Saúde Pública. 2004;20(5):1411-6. 
8. Moretti-Pires RO. O pensamento freireano como superação de desafios do ensino para o SUS. Rev Bras Educ Méd. 2012;36(2):255-63.

9. Mello ALSF, Moysés SJ, Carcereri DL. Ensino ou serviço? A universidade e o curso de Odontologia na rede de atenção à saúde bucal. Mundo saúde. 2011;35(4):364-72.

10. Brasil. Ministério da Saúde. Portaria Interministerial no 1.646, de 5 de agosto de 2014. Institui o componente GraduaCEO - BRASIL SORRIDENTE, no âmbito da Política Nacional de Saúde Bucal, que irá compor a Rede de Atenção à Saúde (RAS), e dá outras providências. Diário Oficial da União, Brasília, 06 de agosto de 2014.

11. Brasil. Ministério da Saúde. Portaria no 4.279, de 30 de dezembro de 2010. Estabelece diretrizes para a organização da Rede de Atenção à Saúde no âmbito do Sistema Único de Saúde (SUS). Diário Oficial da União, Brasília, 30 de dezembro de 2010.

12. Noro LRA, Farias-Santos BCS, Sette-de-Souza PH, Cruz RKS, Pinheiro IAG, Borges REA, et al. $\mathrm{O}$ professor (ainda) no centro do processo ensino-aprendizagem em Odontologia. Rev ABENO. 2015;15(1):2-11.

13. Bardin, L. Análise de conteúdo. Lisboa: Edições 70, 1979.

14. Gonçalves ER, Verdi MIM. Os problemas éticos no atendimento a pacientes na clínica odontológica de ensino. Ciênc Saúde Coletiva. 2007;12(3):755-64.

15. Sponchiado Júnior EC, Souza TB. Estudo da demanda ambulatorial da clínica de odontologia da Universidade do Estado do Amazonas. Ciênc Saúde Coletiva. 2011; 16(1):993-997.

16. Reis SCGB, Santos LB, Leles CR. Clínica integrada de ensino Odontológico: perfil dos usuários e necessidades odontológicas. ROBRAC. 2011;20(4):46-51.

17. Nóbrega LM, Temóteo LM, Dias JN, Lima MSM, Fontes LBC, Cavalcanti SA, et al.
Perception of oral health by patients who use dental clinics. Rev Odontol UNESP. 2013;42(4):259-65.

18. Albuquerque VS, Gomes AP, Rezende CHA, Sampaio MX, Dias OV, Lugarinho RG. A integração ensino-serviço no contexto dos processos de mudança na formação superior dos profissionais da saúde. Rev Bras Educ Méd. 2008;32(3):356-62.

19. Ferreira NP, Dantas TS, Sena-Filho M, Rocha DG. Clínica integrada e mudança curricular: desempenho clínico na perspectiva da integralidade. Pesqui Bras Odontopediatria Clín Integr. 2012;12(1):33-9.

20. Brasil. Ministério da Saúde. Portaria no 1.464, de 24 de junho de 2011. Altera o Anexo da Portaria no 600/GM/MS, de 23 de março de 2006, que institui o financiamento dos Centros de Especialidades Odontológicas (CEO). Diário Oficial da União, Brasília, 27 de junho de 2011.

21. Freitas SFT, Calvo MC, Lacerda JT. Saúde Coletiva e Novas Diretrizes Curriculares em Odontologia: uma proposta para graduação. Trab Educ Saúde. 2012;10(2):223-34.

22. Toassi RFC, Davaglio RS, Lemos VMA. Integração ensino-serviço-comunidade: o estágio na atenção básica da graduação em Odontologia. Educ Rev. 2012;28(4):223-42.

Correspondência para:

Fabiane Alves Farias Guimarães e-mail: fabianealfar@gmail.com 8 Avenue des Coucous Code Postal 44300 - Nantes - France 